\title{
Two-Terminal InGaAs Microwave Amplifier
}

DOI:

10.1002/mop.31261

\section{Document Version}

Accepted author manuscript

Link to publication record in Manchester Research Explorer

\section{Citation for published version (APA):}

Wang, H., Zhang, Y., Shi, Y., Ling, H., Qingpu Wang, Liu, F., Yang, F., Xu, K., Xin, Q., \& Song, A. (2018). TwoTerminal InGaAs Microwave Amplifier. Microwave and Optical Technology Letters, 60, 1877.

https://doi.org/10.1002/mop.31261

\section{Published in:}

Microwave and Optical Technology Letters

\section{Citing this paper}

Please note that where the full-text provided on Manchester Research Explorer is the Author Accepted Manuscript or Proof version this may differ from the final Published version. If citing, it is advised that you check and use the publisher's definitive version.

\section{General rights}

Copyright and moral rights for the publications made accessible in the Research Explorer are retained by the authors and/or other copyright owners and it is a condition of accessing publications that users recognise and abide by the legal requirements associated with these rights.

\section{Takedown policy}

If you believe that this document breaches copyright please refer to the University of Manchester's Takedown Procedures [http://man.ac.uk/04Y6Bo] or contact uml.scholarlycommunications@manchester.ac.uk providing relevant details, so we can investigate your claim.

\section{OPEN ACCESS}




\title{
Two-Terminal InGaAs Microwave Amplifier
}

\author{
Hanbin Wang, Yifei Zhang, Member, IEEE, Yanpeng Shi, Haotian Ling, Qingpu Wang, Fengqi Liu, \\ Fuhua Yang, Kunyuan Xu, Qian Xin and Aimin Song, Senior Member, IEEE
}

\begin{abstract}
The feasibility of using a two-terminal InGaAs planar Gunn structure as a microwave amplifier is proposed and verified. By achieving a pronounced negative differential resistance with a peak-to-valley current ratio of 1.20, our devices are able to amplify microwaves at a high gain of $17 \mathrm{~dB}$. Compared to commonly used three-terminal transistor-based microwave amplifiers, the proposed devices not only have a simple structure but also are capable of achieving high operating frequencies even with relatively large feature sizes. The device with a channel length of $4 \mu \mathrm{m}$ has a positive gain up to about $77 \mathrm{GHz}$, and the $2-\mu \mathrm{m}$ device is able to amplify microwaves well beyond $110 \mathrm{GHz}$. Furthermore, the planar Gunn amplifier shows a good linearity over a wide input power range from -45 to about $0 \mathrm{dBm}$. A good operation stability has also been demonstrated despite having no substrate thinning and heat sink.
\end{abstract}

Index Terms - Gunn amplifiers, Microwave devices, Negative differential resistance, Stability

\section{INTRODUCTION}

$\mathrm{S}_{\mathrm{m}}$ OLID-STATE power amplifiers that operate at microwave and terahertz $(\mathrm{THz})$ frequencies have a wide range of applications, such as radio astronomy, broadband

This work was financed by the National Key Research and Development Program of China (Grant Nos. 2016YFA0301200 and 2016YFA0201800), the National Natural Science Foundation of China (Grant Nos. 11374185, 11304180 and 11404115), Engineering and Physical Sciences Research Council (EPSRC) (Grant No. EP/N021258/1), China Postdoctoral Science Foundation (2015M582073 and 2016M590634), the Natural Science Foundation of Shandong Province (ZR2013EMQ011), the Natural Science Foundation of Jiangsu Province (BK20151255), Suzhou Planning Projects of Science and Technology (SYG201527 and SYG201616), and the Fundamental Research Funds of Shandong University (2013TB008, 2014QY005 and 2016WLJH44). (Corresponding authors: Yifei Zhang and Aimin Song.)

Hanbin Wang, Yifei Zhang, Yanpeng Shi, Haotian Ling, Qingpu Wang, Qian Xin and Aimin Song are with the Center of Nanoelectronics and School of Microelectronics, Shandong University, Jinan 250100, China (e-mail: sduwhb@gmail.com; yifeizhang@sdu.edu.cn; ypshi@sdu.edu.cn; sdulht@gmail.com; wangqingpu@sdu.edu.cn; xinq@sdu.edu.cn; a.song@manchester.ac.uk).

Aimin Song is also with the School of Electrical and Electronics Engineering, University of Manchester, Manchester M13 9PL, U.K.

Fengqi Liu is with the Key Laboratory of Semiconductor Materials Science, Institute of Semiconductors, Chinese Academy of Sciences, Beijing 100083, China (e-mail: fqliu@red.semi.ac.cn).

Fuhua Yang is with the Engineering Research Center for Semiconductor Integration Technology, Institute of Semiconductors, Chinese Academy of Sciences, Beijing 100083, China (e-mail: fhyang@semi.ac.cn).

Kunyuan $\mathrm{Xu}$ is with the Laboratory of Quantum Information Technology \& Engineering Research Center for Optoelectronic Instrument, SPTE, South China Normal University, Guangzhou 510006, China (e-mail: xuky@scnu.edu.cn). wireless communications, radar, medicine imaging, and security inspection [1], [2]. Amplifiers at such high frequencies are typically based on high electron-mobility transistors (HEMTs) on indium phosphide (InP) substrates with channel lengths reduced to tens of nanometers and mushroom-shaped gate [3]. The small channel lengths impose huge challenges in terms of device fabrication and cost, restricting their applications [4]. Though heterojunction bipolar transistors (HBTs) do not rely on nano-scale channels, their high thermal density and sensitivity to high temperatures affect their performance in high-power and heavy load conditions [5]. Prior to the use of transistors, two-terminal devices with negative differential resistance (NDR) in their current-voltage (I-V) characteristics were actually the first solid-state devices for power generation and amplification at microwave frequencies [6]. Although two-terminal amplifiers, such as impact ionization transit-time diodes and resonant-tunneling diodes, have advantages of simple structures and high working frequencies, their impedances are typically strongly dependent on the input power, which limits their high-power applications due to nonlinear distortion [7]-[9]. In contrast, Gunn-diodebased amplifiers show fairly stable impedances and have gained much attention for microwave applications. A Gunn diode may exhibit NDR at high biases due to electrons gaining enough energy to transfer to higher energy valleys within the conduction band. By doing so, the electrons not only lose their kinetic energy to gain higher potential energy but also will have much higher effective mass in the upper valley, hence resulting in a significant reduction of velocity. When biased in the NDR region, the device becomes an active component and microwave signals can be amplified. Compared with other microwave amplifiers, Gunn devices have advantages of simple structure, low cost, high operation frequency, high gain, and low noise [10], [11]. A power gain of $35 \mathrm{~dB}$ has been achieved at a working frequency of $94 \mathrm{GHz}$ [12]. However, the device structure of traditional Gunn devices is vertical, which does not permit efficient fundamental-mode operation at frequency higher than $200 \mathrm{GHz}$ [13]. The Joule heating in vertical Gunn diodes caused by the enormous current density can generate a channel temperature as high as $200{ }^{\circ} \mathrm{C}$ [14]. The high current density and high channel temperature make it very challenging to fabricate suitable ohmic contact. Furthermore, in order to increase the working frequency, the channel length of Gunn device should be reduced, which requires the doping density in the channel to increase proportionally [10]. As such, the heating effect rapidly escalates in short channels, which may easily lead 

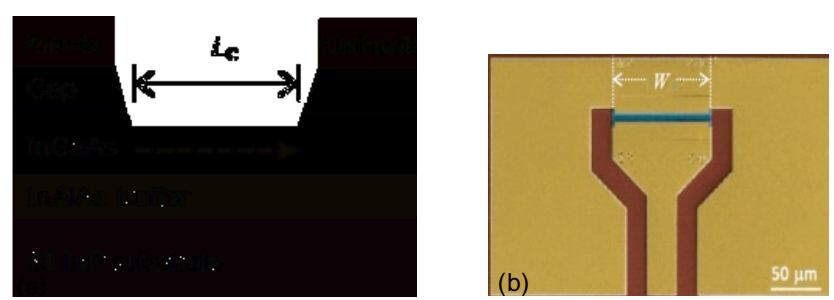

Fig. 1. (a) Cross-section of the InP substrate, and (b) SEM image of a fabricated planar Gunn amplifier with a channel length of $W$.

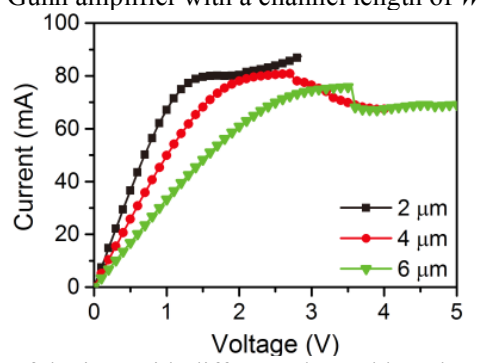

Fig. 2. $I-V$ curves of devices with different channel lengths measured a pulse width of $5 \mathrm{~ms}$.

to breakdown. A number of unusual techniques have to be utilized to overcome this issue, including thinning the substrate down to $2 \mu \mathrm{m}$ and mounting chips on diamond heat sinks, and yet, to the best of our knowledge, the highest fundamental oscillation frequency of InP vertical Gunn diodes is only $160 \mathrm{GHz}$ to date [15]-[17].

In contrast, planar Gunn devices, where the current flows essentially within a very thin two-dimensional (2D) channel, do not suffer a significant Joule heating problem because the current density is much smaller than that in vertical devices, where the current flows through a three-dimensional (3D) bulk channel with a height much smaller than the width. The measured channel temperature of a planar Gunn diodes working in continue wave mode without using any cooling techniques is below $100^{\circ} \mathrm{C}$ [18]. Another advantage of planar structure is that the channel length does not depend on the epitaxial structure of material, but only on the device geometry. This makes it possible to manufacture diodes with different working frequency in one chip. In addition, the planar structure has much smaller parasitic capacitances, which enables higher working frequencies. Progresses of planar Gunn devices have been remarkable in recent years. The fundamental oscillation frequency of planar Gunn diodes has reached $300 \mathrm{GHz}$ [19], which is approximately twice of that of vertical Gunn diodes. Monte Carlo simulation indicates a possible oscillation frequency up to THz region [20], [21], showing a great potential for planar Gunn devices in terahertz applications. However, so far most of planar Gunn devices have been demonstrated only as oscillators. Here, by achieving a pronounced NDR, we are able to show that planar Gunn diode can also be used as a microwave amplifier. Devices with different channel lengths were fabricated and their DC characteristics, microwave amplification performance, and operation stability were studied.

\section{EXPERIMENTAL PROCEDURE}

The planar Gunn amplifiers were fabricated on InGaAs channel layer grown by molecular-beam epitaxy (MBE) on InP
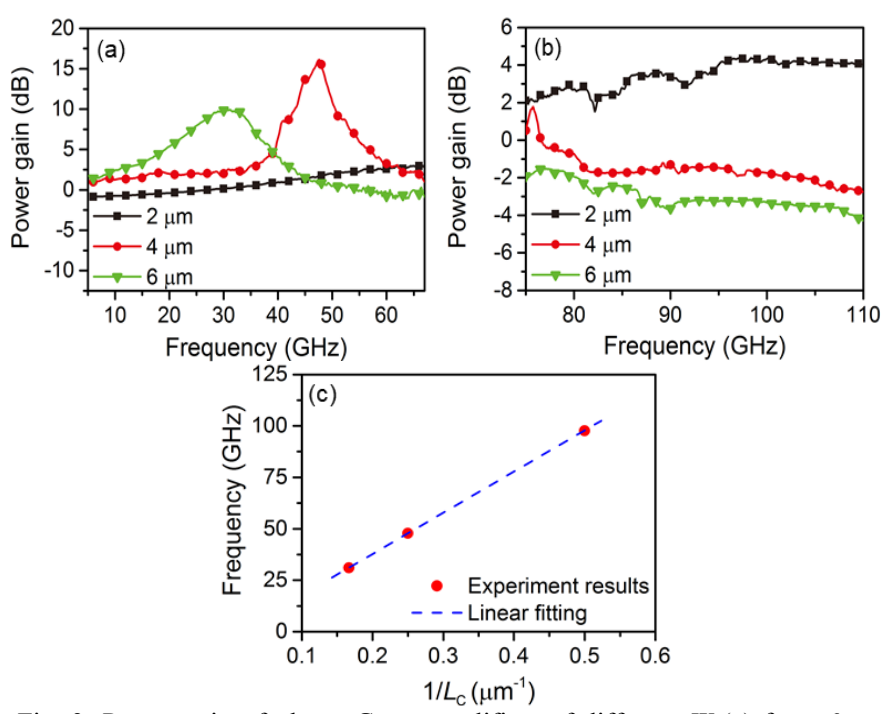

Fig. 3. Power gain of planar Gunn amplifiers of different $W$ (a) from 6 to $67 \mathrm{GHz}$ and (b) from 75 to $110 \mathrm{GHz}$. (c) The peak gain frequency as a function of the reciprocal of channel length. The dashed line is a linear fit.

wafer including a $300 \mathrm{~nm} \operatorname{In}_{0.52} \mathrm{Al}_{0.48}$ As buffer layer, a $300 \mathrm{~nm}$ InGaAs channel layer, and a $200 \mathrm{~nm}$ InGaAs cap layer. The doping density $\left(N_{\mathrm{D}}\right)$ of the channel layer is $8 \times 10^{16} \mathrm{~cm}^{-3}$ to make sure $N_{\mathrm{D}} L_{\mathrm{C}}>10^{12} \mathrm{~cm}^{-2}$ [22]. The cap layer was doped at $2 \times 10^{18} \mathrm{~cm}^{-3}$. Fabrication started with wet etching to define mesa and channel. As shown in Fig. 1(a), three different channel lengths $\left(L_{\mathrm{C}}\right)$, i.e., 2,4 , and $6 \mu \mathrm{m}$, were investigated. The Ohmic contact, consisting of $\mathrm{Pd} / \mathrm{Ge} / \mathrm{Au} / \mathrm{Ti} / \mathrm{Au}$, was deposited using electron-beam evaporation. The contact resistance is $0.35 \Omega \cdot \mathrm{mm}$. Finally, co-planar waveguide (CPW) was formed using evaporated $\mathrm{Ti}$ and $\mathrm{Au}$. A colored scanning-electron microscope image of device is shown in Fig. 1(b). The width of channel $(W)$ is $100 \mu \mathrm{m}$. All patterns were defined by electronbeam lithography (EBL) with Poly (methyl methacrylate) (PMMA, molecular weight 950K) resist.

\section{Results AND Discussion}

Similar to other types of two-terminal amplifiers, Gunndevice-based amplification is based on negative differential conductance (NDC, the reciprocal of NDR) [23]. As shown in Fig. 2, pronounced NDC can be observed in the $I-V$ characteristics of planar Gunn amplifiers measured in pulsed mode. The pulse width is $5 \mathrm{~ms}$, and the period is $200 \mathrm{~ms}$. The peak NDC of devices with $L_{\mathrm{C}}=4$ and $6 \mu \mathrm{m}$ is $-32.2 \mathrm{mS}$ and $-156 \mathrm{mS}$, respectively. The device with $L_{\mathrm{C}}=2 \mu \mathrm{m}$ shows a much smaller NDC than the longer devices, and its current starts to increase soon after the NDR region appears at about $1.5 \mathrm{~V}$. This should be mainly attributed to a relatively large series resistance in the short-channel device [24]. The measured series resistance is $4 \Omega$, which accounts for $30 \%$ of the total resistance of the 2- $\mu \mathrm{m}$ device. Thus, NDC will be reduced or totally vanished by large series resistance [25], [26].

Current peak-to-valley ratio (PVR) is one of the key parameters of NDR-based amplifying devices that determines the DC to RF conversion efficiency $(\eta)$. The relationship can be 
expressed as

$$
\eta=\frac{(\mathrm{PVR}-1)(\beta-1)}{(\mathrm{PVR}+1) \beta}\left(\frac{8}{\pi^{2}}\right),
$$

where $\beta$ is the ratio of $\mathrm{DC}$ bias to threshold voltage [27]. The measured PVR of the $4 \mu \mathrm{m}$ device is 1.20 . To the best of our knowledge, this is the highest measured PVR in InGaAs planar Gunn devices with similar dimensions. The high PVR may be attributed to the InAlAs buffer layer and high quality of the semiconductor layers epitaxially grown by MBE. The buffer provides a high degree of layer homogeneity and high-quality interfaces with fewer electron scattering under high electric field so that the electrons can obtian high energy to transfer to the higher energy valley, inducing a pronounced NDR.

RF characteristics of planar Gunn amplifiers were investigated from 6 to $110 \mathrm{GHz}$ using a vector network analyzer (VNA). VNA generates a RF signal to the device under test and measures the ratio of the reflect power to the input, i.e., power gain of two-terminal devices. Figures 3(a) and (b) present power gain of the fabricated devices with different channel lengths. All devices show good power amplification performance. The maximum power gain of devices with $L_{\mathrm{C}}=6$, 4 , and $2 \mu \mathrm{m}$ is $10 \mathrm{~dB}$ at $31.0 \mathrm{GHz}, 15 \mathrm{~dB}$ at $47.8 \mathrm{GHz}$, and $4.5 \mathrm{~dB}$ at $97.6 \mathrm{GHz}$, respectively, and their $3-\mathrm{dB}$ bandwidth is 12.6, 5.2, and $35.0 \mathrm{GHz}$, respectively. Power gain and band width of the devices can be further improved by using matching networks [28].

It can be seen in Figs. 3(a) and (b) that devices with short channels can work at high frequencies. In order to reveal the relationship between the frequency of peak gain and channel length, the peak frequency is plotted as a function of the reciprocal of the channel length in Fig. 3 (c). The dotted and dashed lines represent the measured results and a linear fitting, respectively. Their good agreement indicates an expected linear dependence. The slope of the fitted line is $2.0 \times 10^{5} \mathrm{~m} / \mathrm{s}$, which matches well with the room-temperature saturation drift velocity of InGaAs [29]. This is consistent with the relationship between oscillation frequency and channel length in planar Gunn diode [25]. Based on this, the working frequency of planar Gunn amplifiers can be predicted to reach $300 \mathrm{GHz}$, if the channel length is reduced to $0.6 \mu \mathrm{m}$, i.e., about ten times that of HEMT amplifiers at the same frequency [30]. This significantly eases the challenges to fabricate extremely small HEMT based microwave amplifiers, particularly for upper band of microwaves and terahertz signals.

Figure 4(a) shows the relationship between the power gain and input signal power. Measurements were performed on a 4- $\mu \mathrm{m}$ amplifier at $45 \mathrm{GHz}$ under biases of 2.9 and 4.0 V. Both curves show good power stabilities with input power up to $-5 \mathrm{dBm}$. The input power level at which the gain drops by $1 \mathrm{~dB}$ from the small signal value is commonly defined as $1-\mathrm{dB}$ gain compression point. The $1-\mathrm{dB}$ compression point of device is $0 \mathrm{dBm}$ at $2.9 \mathrm{~V}$ with a power gain of $7 \mathrm{~dB}$, and $-5 \mathrm{dBm}$ at $4.0 \mathrm{~V}$ with a power gain of $13 \mathrm{~dB}$. These are comparable to the previously published vertical Gunn amplifiers [11], [31]. The dashed line rectangles in Fig. 4(a) represent the linear amplifying regions.
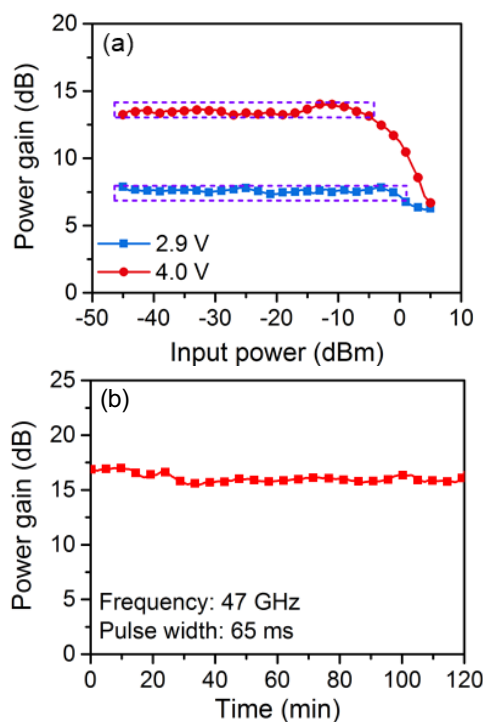

Fig. 4. (a) Measured power gain of planar Gunn amplifier as a function of input signal power with different DC biases. (b) Stability test of a $4-\mu \mathrm{m}$ device at room temperature for two hours.

Thermal stability is another important issue to evaluate power amplifiers. Figure 4(b) shows the power gain of a $4 \mu \mathrm{m}$ device measured at $47 \mathrm{GHz}$ in pulse mode for two hours with pulse width and pulse period is $65 \mathrm{~ms}$ and $2 \mathrm{~s}$, respectively. The average power gain is $16 \mathrm{~dB}$ with fluctuation less than $0.8 \mathrm{~dB}$. Considering that no cooling technique has been applied, this result indicates that the planar Gunn amplifier is much more robust than the traditional vertical Gunn devices in which substrate thinning and heat sink are common necessities.

\section{CONCLUSION}

In summary, two-terminal InGaAs planar Gunn amplifiers have been demonstrated for the first time. Pronounced NDR and high power gain were observed in 4 and $6 \mu \mathrm{m}$ devices. Operating frequency up to $110 \mathrm{GHz}$ were realized in the device with a channel length of $2 \mu \mathrm{m}$. The experimental results indicate that the peak-gain frequency is inversely proportional to the channel length. Despite having no substrate thinning, heat sink, and other cooling conditions, the amplifiers show robust thermal stability. Due to the advantages of simple fabrication, low cost, high frequency, high gain, and wide linear-power-gain range, the two-terminal amplifiers may find useful applications at microwave and terahertz frequencies.

\section{REFERENCES}

[1] A. Rostami, H. Rasooli, and H. Baghban, Terahertz Technology Fundamentals and Applications vol. 77. New York: Springer-Verlag Berlin Heidelberg, 2011.

[2] W. Withayachumnankul, G. M. Png, et al., "T-Ray Sensing and Imaging," in Proc. of the IEEE, vol. 95, pp. 1528-1558, 2007.

[3] L. A. Samoska, "An Overview of Solid-State Integrated Circuit Amplifiers in the Submillimeter-Wave and THz Regime," IEEE Transactions on Terahertz Science and Technology, vol. 1, pp. 9-24, 2011.

[4] C. T. Coen, A. C. Ulusoy, et al., "Design and On-Wafer Characterization of G -Band SiGe HBT Low-Noise Amplifiers," IEEE Transactions on Microwave Theory and Techniques, vol. 64, pp. 3631-3642, 2016. 
[5] W. Liu, C. Hin-Fai, and E. Beam, "Thermal properties and thermal instabilities of InP-based heterojunction bipolar transistors," IEEE Transactions on Electron Devices, vol. 43, pp. 388-395, 1996.

[6] G. I. Haddad, J. R. East, and H. Eisele, "Two-Terminal Active Devices for Terahertz Sources," International Journal of High Speed Electronics and Systems, vol. 13, pp. 395-427, 2003.

[7] H. Komizo, Y. Ito, H. Ashida, and M. Shinoda, "A 0.5-W CW IMPATT diode amplifier for high-capacity 11-GHz FM radio-relay equipment," IEEE Journal of Solid-State Circuits, vol. 8, pp. 14-20, 1973.

[8] D. C. Hanson and W. W. Heinz, "Integrated electrically tuned X-band power amplifier utilizing Gunn and IMPATT diodes," IEEE Journal of Solid-State Circuits, vol. 8, pp. 3-14, 1973.

[9] M. E. Hines, "Negative-resistance diode power amplification," IEEE Transactions on Electron Devices, vol. 17, pp. 1-8, 1970.

[10] H. Eisele, "Gunn or Transferred-Electron Devices," Wiley Encyclopedia of Electrical and Electronics Engineering, pp. 1-19, 2014.

[11] A. Sene and F. J. Rosenbaum, "A Wide-Band Gunn-Effect CW Waveguide Amplifier," IEEE Transactions on Microwave Theory and Techniques, vol. 20, pp. 645-650, 1972.

[12] L. J. Messick, B. Fank, et al., "Basics And Recent Applications of High Efficiency Millimeter Wave InP Gunn Diodes," in 1st Int. Conf. on Indium Phosphide and Related Materials for Advanced Electronic and Optical Devices, Norman, America, 1989, pp. 534-546.

[13] A. Khalid, N. J. Pilgrim, et al., "A Planar Gunn Diode Operating Above 100 GHz," IEEE Electron Device Letters, vol. 28, pp. 849-851, 2007.

[14] M. A. di Forte-Poisson, G. Colomer, et al., "High Power, High Efficiency LP. MOCVD InP Gunn Diodes for 94GHz," in 15th European Microwave Conf., pp. 177-182, 1985.

[15] R. Kamoua, H. Eisele, and G. I. Haddad, "D-band (110-170 GHz) InP gunn devices," Solid-State Electronics, vol. 36, pp. 1547-1555, 1993.

[16] H. Eisele, A. Rydberg, and G. I. Haddad, "Recent advances in the performance of InP Gunn devices and GaAs TUNNETT diodes for the 100-300-GHz frequency range and above," IEEE Transactions on Microwave Theory and Techniques, vol. 48, pp. 626-631, 2000.

[17] H. Eisele and R. Kamoua, "Submillimeter-Wave InP Gunn Devices," IEEE Transactions on Microwave Theory and Techniques, vol. 52, pp. 2371-2378, 2004.

[18] M. Ismaeel Maricar, J. Glover, et al., "Planar gunn diode characterisation and resonator elements to realise oscillator circuits," in 2013 Int. Conf. on Advanced Nanomaterials and Emerging Engineering Technologies (ICANMEET), Chennai, 2013.

[19] A. Khalid, G. M. Dunn, et al., "Terahertz oscillations in an In0.53Ga0.47As submicron planar Gunn diode," Journal of Applied Physics, vol. 115, p. 114502, 2014.

[20] S. Pérez, T. González, D. Pardo, and J. Mateos, "Terahertz Gunn-like oscillations in InGaAs/InAlAs planar diodes," Journal of Applied Physics, vol. 103, p. 094516, 2008.

[21] K. Y. Xu, G. Wang, and A. M. Song, "Gunn oscillations in a selfswitching nanodiode," Applied Physics Letters, vol. 93, p. 233506, 2008.

[22] Ó. García-Pérez, Y. Alimi, et al., "Experimental assessment of anomalous low-frequency noise increase at the onset of Gunn oscillations in InGaAs planar diodes," Applied Physics Letters, vol. 105, p. 113502, 2014.

[23] H. Eisele and G. I. Haddad, "Two-terminal millimeter-wave sources," IEEE Transactions on Microwave Theory and Techniques, vol. 46, pp. 739-746, 1998.

[24] B. K. Ridley and T. B. Watkins, "The Possibility of Negative Resistance Effects in Semiconductors," Proceedings of the Physical Society, vol. 78, pp. 293-304, 1961.

[25] C. Li, A. Khalid, et al., "Design, fabrication and characterization of $\mathrm{In}_{0.23} \mathrm{Ga}_{0.77} \mathrm{As}$-channel planar Gunn diodes for millimeter wave applications," Solid-State Electronics, vol. 64, pp. 67-72, 2011.

[26] M. Masuda, et al., "Bistable switching in a planar Gunn diode," IEEE Transactions on Electron Devices, vol. 25, pp. 358-361, 1978.

[27] S. M. Sze and K. K. Ng, Physics of Semiconductor Devices. New Jersey: John Wiley \& Sons, 2006.

[28] R. J. Hamilton, R. D. Fairman, et al., "InP Gunn-Effect Devices for Millimeter-Wave Amplifiers and Oscillators," IEEE Transactions on Microwave Theory and Techniques, vol. 24, pp. 775-780, 1976.

[29] S. Adachi, Physical Properties of III-V Semiconductor Compounds: InP, InAs, GaAs, GaP, InGaAs, and InGaAsP. New York: John Wiley \& Sons, 1992.

[30] X. Mei, W. Yoshida, et al., "First Demonstration of Amplification at 1 THz Using 25-nm InP High Electron Mobility Transistor Process," IEEE Electron Device Letters, vol. 36, pp. 327-329, 2015.
[31] H. W. Thim, "Linear microwave amplification with Gunn oscillators," IEEE Transactions on Electron Devices, vol. 14, pp. 517-522, 1967. 